\title{
Interactive comment on "An
}

\section{observationally-constrained estimate of global dust aerosol optical depth" by David A. Ridley et} al.

David A. Ridley et al.

daridley@mit.edu

Received and published: 27 October 2016

\section{Response to Reviewers}

We would like to thank all reviewers for their helpful comments and criticism on this work. We believe we have addressed the comments and made changes to the methodology and manuscript where possible. We now include supplementary figures and several of the figures in the manuscript have been updated.

Key changes include:

âĂć Analysis and statistics generated for $\log (A O D)$ rather than AOD 
âĂć Instrument uncertainty included in the estimate

âÁć Regional bias correction of satellite data by AERONET

âĂć Uncertainty in bias correction propagated through analysis

âĂć Marine Aerosol Network (MAN) data included

Interactive

comment

âĂć Supplementary figures of AERONET and satellite AOD histograms

âÁć Comparison of model AOD with daily AOD from MAN

âÁć Supplementary comparison with deposition flux

âĂć

The key changes are that the global dust AOD is decreased from 0.033 to 0.030 and the uncertainty increased from 0.006 to $0.011(2 \sigma)$ as a result of considering instrument uncertainty and the uncertainty on the updated AERONET bias correction of the satellite retrievals. The observational estimate is hence closer to the AEROCOM model estimate. We believe that this better corrects for regional biases in the satellite retrievals while representing the inherent uncertainty in using limited in-situ measurements to apply correction factors over large regions. The regional estimates of seasonal dust AOD from the different satellite instruments are generally in closer agreement. The observational estimate is also brought closer to the MERRAero dust AOD; the previous discrepancy was of some concern because MERRAero assimilates MODIS AOD and may be expected to represent the dust AOD better than models without assimilation. The agreement between model and observational estimate improves over the mid-Atlantic, reducing (but not eliminating) the potential for systematically high dust removal in the models. While many of the quoted numbers change as a result of our reanalysis, all other conclusions remain essentially the same.

Printer-friendly version

Please find the reviewer-specific comments and responses listed below.

Discussion paper

Kind regards, David Ridley 
Amato Evan This manuscript describes a method of combining satellite and model data in order to estimate the global dust AOD (DAOD). The principal idea here is that models do a good job of simulating non-dust AOD, and satellites do a good job of retrieving the total AOD, so the difference between the two should be a good estimate of DAOD. While I applaud the authors on their creative effort, and the obviously massive amount of time undertaken to complete this work, I find there to be a couple of major issues with the methods that I suspect are contributing to a bias in their global DAOD estimate, and increase the uncertainty. Thus, I am suggesting a major revision.

Major Comments 1. A major assumption of this method is that model DAOD is biased, but that model AOD is not. However, this assumption, at least the part about model AOD not having any systematic bias, isn't justified. The authors suggest that they are accounting for errors related to underestimation of the non-dust AOD by reporting their global DAOD with a 2-sigma uncertainty range (P13, L15). However, if the models systematically underestimate the non-dust AOD, this will induce a high bias in their reported global DAOD, and thus simply increasing the uncertainty range isn't really appropriate. We need to know if there is a bias, particularly because a low bias in modeled non-dust AOD would serve to push the hybrid global DAOD estimate closer to the aerocom mean, and possible closer to the MERRAaero estimate. One could determine if such a bias exists by comparing histograms of AOD for the models and AERONET, over land regions and over-water regions where there is no dust (but there is smoke, anthro. aerosols, and marine aerosols). The difference in those histograms can be used to calculate a bias (which could be corrected) and uncertainty in the models' non-dust AOD. These errors can then be carried through to the final global DAOD calculation.

$>$ A major assumption of this method is that model DAOD is biased, but that model AOD is not. However, >this assumption, at least the part about model AOD not having any systematic bias, isn't justified. 
This is certainly a valid concern. We use multiple models to estimate the uncertainty and consider regions where dust aerosol dominates the AOD to minimize the impact of errors in modeled non-dust AOD. For example, the Gulf of Guinea region is not considered explicitly because of the influence of biomass burning. However, as you point out, this may not be sufficient if all models are biased in the same direction. The difficulty is in isolating cases in the dust-influenced regions we consider, but when the dust AOD is not significant. For example, even if we look during wintertime in the Middle East, when dust emissions are low, there is no guarantee that the non-dust AOD will adequately represent the non-dust present during the summertime (looking in other regions, as suggested, is problematic as we may just be observing a local bias that is irrelevant to the dusty locations).

To explore potential biases in modeled non-dust AOD we separate the daily coincident AERONET and model AOD based on whether the model dust AOD contributes $>60 \%$ or $<60 \%$ of the total AOD. There is indeed evidence of a bias in CESM and GEOSChem. From the added supplementary materials:

"We find that there is a bias between these two cases where CESM and GEOS-Chem both underestimate the AOD relative to AERONET in low dust cases and overestimate the AOD in high dust cases. WRF-Chem and MERRAero show a smaller bias in the opposite direction. Relative to AERONET, the models are biased by $-23 \%,-20 \%,+3 \%$, $+10 \%$ (GEOS-Chem, CESM, WRF-Chem and MERRAero, respectively) for the low dust cases, and biased $+33 \%,+12 \%,+14 \%$ and $+6 \%$ for the high dust cases. The days with low dust AOD in the models are biased low most at AERONET sites in the Thar Desert and Kyzyl Kum, that have limited AERONET data, in the Middle East, and across Africa. This suggests that the non-dust AOD in the models may be biased low on average, which would lead to a high bias in the observational estimate of the dust AOD.

If we re-run the analysis including a regional bias correction factor for the models, we find that the mean estimate of the global dust AOD is reduced to 0.028 , a $7 \%$ decrease

Printer-friendly version

Discussion paper
Interactive

comment

\section{C4}


but still well within our uncertainty estimate. However, with the bias correction applied, the observational estimate of dust AOD in the Mid-Atlantic is unrealistically close to zero in winter and can end up being consistently negative in the Thar desert, suggesting the bias correction is overcompensating. The agreement in seasonal dust AOD between different satellite-model realizations is also worsened, rather than improved. Finally, there is no guarantee that the model dust AOD is an adequate filter to partition the data into low/high dust days, the filter may simply select for seasons when less dust present, which might not tell us much about the non-dust AOD in seasons when dust is present. For this reason, we do not bias-correct the model non-dust AOD for the observational estimate of global dust AOD presented in this work. However, we highlight this potential source of uncertainty in the main text and included a reference to this supplementary text in the summary of explored biases and uncertainty (Table 2)."

In the main text (Section 4.4, pg 15) we add:

"...the non-dust AOD in all models may be systematically biased high or low, which would bias the observational estimate of the dust AOD low or high, respectively. Comparison between modeled and observed AOD at the AERONET sites and MAN ship locations does suggest a low bias in the modeled total AOD in some of the regions considered, although there is no clear systematic bias in the models (see Figures S5 S9). Comparison of model and AERONET AOD in low and high dust cases (using the model dust AOD to discriminate) suggests that two of the models are biased high and two biased low (Figure S4). Overall, the ensemble of models appears to underestimate the non-dust AOD; correcting this results in a 7\% decrease in the global dust AOD estimate (0.028). However, the uncertainties involved in this method are such that we do not include the bias correction in our final estimate (see Supplementary Materials)."

2. I am also very concerned about use of the models' spatial structure of DAOD (the 
horizontal pattern of long-term mean DAOD). In Eqn 2 the authors rely on the spatial structure of modeled DAOD in order to estimate their hybrid global DAOD. The implicit assumption is that while the models' may exhibit biases in the absolute value of DAOD, they do a good job of reproducing the long-term mean spatial structure. However, later on in the paper (P11, Section 4.3) the authors examine the signs of the difference between modeled DAOD and that from their hybrid method in Fig 9 (Africa, N Atl, Gulf of Guinea), suggesting that the models emit too much dust at the source to compensate for the fact that wet and dry deposition is far too strong. So on the one hand you are saying that the spatial structure of model DAOD is good (Eqn 2) and on the other hand it's not (Fig 9). If your hypothesis is correct, that the models emit too much dust because deposition is too strong, then Eqn 2 will introduce a bias into your global DAOD estimate depending on the relative fraction of regions (Fig 1 ) that are over dust emitting areas and those that are downwind. I think this means that because your regions in Figure 1 are overwhelmingly near or over dust sources, your final global DAOD estimate could be biased low? I'm not entirely sure. . . But the bottom line is that, given this bias in the spatial structure of dust from the models, there is an additional source of uncertainty in the global DAOD estimate, and potentially a bias, related to the distribution of the regions you choose (Fig 1). I'm not exactly sure how you can address this. Maybe add more over-water regions and redo the estimate only using over-water regions, the only using over-land regions, then using both (via Eqn 2)? Or maybe the way to address this potential bias/uncertainty is to recalculate global DAOD using an equal distribution of regions over dust sources and regions downwind of dust sources (also in Eqn 2).

Yes, this is a fair point. The short answer is that there is could be a bias, assuming the excessive removal we infer in the Atlantic is a global issue, but comparison of satellite and model DAOD in remote locations is not possible. We were unable to use the bootstrapping method to determine satellite DAOD over remote regions simply because the DAOD is too low relative to other aerosol AOD and the retrieval sensitivity is too weak (we compared the satellite-retrieved AOD to the MAN network and found that the agreement is much poorer and of limited use over remote locations in the 
We compare the models with the MAN observations in remote locations to see if there is an obvious low bias. While correlation is poor in remote regions, there is no clear systematic bias present in the models. However, it is not clear how much of a role dust versus non-dust aerosol plays in this. We refer to previous comparisons of modeled and observed dust surface concentration that show considerable spread in the agreement in remote regions (see Figure 4 of Huneeus et al.; 2011) that limit our ability to discern whether the models used here are unbiased in their representation of the localto-remote dust distribution. We have added the supplementary figures of comparison of AOD between models and AERONET and MAN, and in the manuscript we have more clearly highlighted this uncertainty in Section 4.4 ("Discussion of the remaining uncertainties"):

"Modeled dust AOD is used as a scaling factor to determine the global dust AOD from the regional observational estimates. We use multiple models to represent the uncertainty, but there may be a systematic bias present, rather than the $\pm 6 \%$ uncertainty presented (Table 2). If the over-zealous removal of dust in models, highlighted in the mid-Atlantic, is a global phenomenon then the models would predict too much dust in the source regions relative to downwind and yield a low regional-to-global scaling factor. Similarly, dust emissions schemes currently used in the models are unlikely to reproduce emissions where vegetation cover is variable and will not represent dust from agricultural regions (Ginoux et al., 2012). If those emissions are substantial, then it is possible that tuned emissions in models overestimate emissions from large, permanent dust sources to compensate for the lack of agricultural emissions, which could partially explain model bias towards African emissions.

3. Lastly, I think models report AOD even in the presence of $100 \%$ cloud cover. So, in the model, there could be an aerosol layer overlaying stratus clouds, and the model 
would save an AOD value. However, in the satellite world, there would be no AOD retrieval. Does this discrepancy induce a bias? Can you examine the model data (I guess you'd need daily or hourly output) to see?

Yes, we have performed this analysis to compare the DAOD with all GEOS-Chem data and with the model data masked where any grid box in a column has $>50 \%$ cloud cover (based on MERRA reanalysis). We find that the change from masking is small, resulting in a $2 \%$ increase in the DAOD. We now mention this explicitly in the main text (pg 11):

"We also calculated GEOS-Chem global dust AOD after masking columns that have $>50 \%$ cloud cover in any grid box, based on MERRA reanalysis. This causes the global dust $A O D$ to increase by $2 \%$, relative to when no masking is used, indicating that the difference between clear-sky and all-sky dust AOD is small. However, we acknowledge that poor representation of clouds in the reanalysis meteorology or potential satellite misclassification of heavy dust loading as cloud (Darmenov and Sokolik, 2009) could lead to a stronger perceived relationship between dust loading in cloudy and clear sky conditions."

also by masking the model AOD with satellite retrievals. The latter effectively masks for clouds as well as for overpass frequency for better comparison between model and observations. Masking the model DAOD with Aqua and Terra has a negligible effect on the global DAOD $(<1 \%)$ and masking with MISR increases the global DAOD by $1-2 \%$. This rather surprising finding indicates that the model global DAOD is not significantly different whether or not cloudy locations are included. We have made this more explicit in the text as follows:

"We calculate the modeled global dust AOD with and without masking to match the MODIS and MISR sampling, testing whether sampling affects the derived global dust AOD. We find negligible $(<1 \%)$ changes in the modelled global dust AOD when sampling to the MODIS instruments and an increase of $1-2 \%$ when sampling to MISR. 
Therefore, we determine that sampling frequency is sufficient to represent the AOD in the regions considered. Furthermore, because the masking effectively removes cloudy regions, the very small change in the modelled global dust AOD indicates that there is no obvious bias in the global dust AOD when including regions within cloudy air masses, relative to clear-sky only"

Minor Comments 1. P7, L4: Spelling, "main" not "man" Changed

2. Should alpha have a region superscript in Eqn 1? Previously no, but now that the bias correction is regional it has been added.

3. P11, L25: Why would a lack of convectively driven dust emissions cause an overestimation of DAOD? Seems like it would be the opposite. The convectively driven dust is strongest in summer, so a lack of that source causes an over-emphasis of winter and spring relative to summer. I think the sentence reflects your intuition.

4. P7, L26: You write, "In the regions analyzed here the AOD is predominantly driven by dust aerosol, limiting the influence of the model non-dust AOD" but this simply isn't true. Region 1 (N. Atl) also has a big biomass burning contribution in the boreal winter. Regions 8 also has a contribution from anthro. aerosols from N. India during the dry monsoon season. Same for region 10 (from Pakistan and Iran).

Yes, this statement does overemphasize the importance of dust aerosol. We have softened the language as follows:

"In the regions analyzed here dust aerosol plays a key role and often dominates in the spring and summer, limiting the influence of the model non-dust AOD. Exceptions 
to this are in South America, South Africa, and Australia, that have a minimal impact on the global dust AOD, and the Gulf of Guinea, where significant biomass burning aerosol is present (we consider results with and without these regions, see Table 1)."

5. P10: Cloud filtering: Interesting that you are getting such a strong correlation between the two. Misclassification of optically thick dust as cloud may be pretty common, FYI.

Yes, we thought this was interesting. We have added a reference to Darmenov and Sokolik (2009) in there to point out that misclassification of optically thick dust may be occurring.

"We acknowledge that satellite misclassification of heavy dust loading as cloud may occur (Darmenov and Sokolik, 2009) potentially leading to a stronger relationship between dust loading in cloudy and clear sky conditions."

Interactive comment on Atmos. Chem. Phys. Discuss., doi:10.5194/acp-2016-385, 2016. 